\title{
Expression of non-homologous end joining factor, Ku80, is negatively correlated with PD-L1 expression in cancer cells after X-ray irradiation
}

\author{
TAKUYA KUMAZAWA ${ }^{1,2^{*}}$, YASUMASA MORI ${ }^{1,3^{*}}$, HIRO SATO $^{1}$, TIARA BUNGA MAYANG PERMATA ${ }^{4}$, \\ YUKI UCHIHARA $^{5}$, SHIN-EI NODA ${ }^{6}$, KOHEI OKADA ${ }^{1}$, SANGEETA KAKOTI $^{1,5,7}$, KEIJI SUZUKI $^{8}$, \\ HAYATO IKOTA $^{9}$, HIDEAKI YOKOO ${ }^{10}$, SOEHARTATI GONDHOWIARDJO ${ }^{4}$, TAKASHI NAKANO ${ }^{3}$, \\ TATSUYA OHNO $^{1}$ and ATSUSHI SHIBATA ${ }^{5}$
}

\begin{abstract}
${ }^{1}$ Department of Radiation Oncology, Graduate School of Medicine, Gunma University, Maebashi, Gunma 3718511;
${ }^{2}$ Department of Radiation Oncology, Saku Central Hospital Advanced Care Center, Saku, Nagano 3850051;

${ }^{3}$ National Institute of Radiological Sciences, National Institute for Quantum and Radiological Science and Technology,

Chiba 2638555, Japan; ${ }^{4}$ Department of Radiation Oncology, Faculty of Medicine Universitas Indonesia,

Dr Cipto Mangunkusumo National General Hospital, Jakarta 10430, Indonesia; ${ }^{5}$ Signal Transduction Program, Gunma University Initiative for Advanced Research, Gunma University, Maebashi, Gunma 3718511; ${ }^{6}$ Department of

Radiation Oncology, Comprehensive Cancer Centre, International Medical Centre, Saitama Medical University, Saitama 3501298, Japan; 7 Department of Radiation Oncology, Tata Memorial Centre, Homi Bhabha National Institute, Mumbai, Maharashtra 400012, India; ${ }^{8}$ Department of Radiation Medical Sciences, Atomic Bomb Disease Institute, Nagasaki University, Nagasaki 852-8523; ${ }^{9}$ Clinical Department of Pathology, Gunma University Hospital; ${ }^{10}$ Department of Human Pathology, Gunma University Graduate School of Medicine, Maebashi, Gunma 3718511, Japan
\end{abstract}

Received May 13, 2021; Accepted October 14, 2021

DOI: 10.3892/ol.2021.13147

\begin{abstract}
The growing importance of antitumour immunity by cancer immunotherapy has prompted studies on

Correspondence to: Professor Tatsuya Ohno or Dr Hiro Sato, Department of Radiation Oncology, Graduate School of Medicine, Gunma University, 3-39-15 Showa, Maebashi, Gunma 3718511, Japan E-mail: tohno@gunma-u.ac.jp

E-mail: hiro.sato@gunma-u.ac.jp

*Contributed equally
\end{abstract}

Abbreviations: BRCA, breast invasive carcinoma; CESC, cervical squamous cell carcinoma and endocervical adenocarcinoma; COAD, colon adenocarcinoma; DSB, double-strand break; HNSC, head and neck squamous cell carcinoma; ICI, immune checkpoint inhibitors; LUAD, lung adenocarcinoma; MFI, mean fluorescence intensity; NHEJ, non-homologous end joining; SKCM, skin cutaneous melanoma; STING, stimulator of IFN genes; TCGA, The Cancer Genome Atlas; TPS, tumour proportion score; UCEC, uterine corpus endometrial carcinoma; ATR, ataxia telangiectasia and Rad3-related; Chk1, checkpoint kinase 1; DNA-PKcs, DNA-dependent protein kinase, catalytic subunit; XRCC4, X-ray repair cross-complementing protein 4; LIG4, DNA ligase 4

Key words: radiotherapy, immunotherapy, DNA damage response, programmed death-1 ligand, Ku80, biomarker radiotherapy-induced immune response. Previous studies have indicated that programmed cell death-1 ligand (PD-L1) expression is regulated by DNA damage signalling. However, PD-L1 up-regulation after radiotherapy has not been fully investigated at the clinical level, particularly in the context of expression of DNA repair factors. The present study examined the correlation of mRNA expression between PD-L1 and non-homologous end joining (NHEJ) factors using The Cancer Genome Atlas database analysis. Among NHEJ factors, $K u 80 \mathrm{mRNA}$ expression was negatively correlated with $P D-L 1$ mRNA expression levels in several types of cancer (colon adenocarcinoma, breast invasive carcinoma, skin cutaneous melanoma, lung adenocarcinoma, head and neck squamous cell carcinoma, uterine corpus endometrial carcinoma, cervical squamous cell carcinoma and endocervical adenocarcinoma). To verify the negative correlation in clinical samples, the present study analysed whether Ku80 expression levels affected PD-L1 up-regulation after radiotherapy using cervical squamous cell carcinoma samples. Quantitative evaluation using software analysis of immunohistochemically stained slides revealed that patients with low Ku80 positivity in biopsy specimens demonstrated increased PD-L1 expression levels after $10 \mathrm{~Gy}$ irradiation (Spearman's rank correlation coefficient $=-0.274 ; \mathrm{P}=0.017)$. Furthermore, PD-L1 induction levels in tumour cells after $10 \mathrm{~Gy}$ of irradiation were significantly inversely correlated with Ku80 expression levels (Spearman's rank correlation coefficient=-0.379; $\mathrm{P}<0.001$ ). The present study also confirmed that short interfering 
RNA-mediated Ku80 depletion was associated with greater X-ray-induced PD-L1 up-regulation in HeLa cells. These results indicated that radiotherapy could enhance PD-L1 induction in tumour cells with low Ku80 expression in a clinical setting. Furthermore, these data highlighted Ku80 as a potential predictive biomarker for immune checkpoint therapy combined with radiotherapy.

\section{Introduction}

The success of immune checkpoint inhibitors (ICIs) targeting programmed cell death-1 (PD-1)/programmed cell death-1 ligands (PD-L1) and cytotoxic T lymphocyte-associated antigen-4 (CTLA-4) has highlighted the important role of antitumour immunity in cancer treatment (1). In recent years, there has been increased interest in studies on antitumour immunity for effective cancer treatment (2). Clinically, a combination strategy of ICIs and other cancer therapies that induce local and systemic immune responses achieve an improved response rate compared with ICIs alone $(3,4)$.

Previous studies have suggested stimulation of local and systemic immune responses after radiotherapy; for example, radiotherapy promotes the induction of damage-associated molecular patterns and tumour-associated antigens as well as the release of exosomes containing DNA fragments that activate dendritic cells (5-7). In addition, radiotherapy up-regulates the expression of human leukocyte antigen, a central component of specific immune stimulation (8). Increased serum levels of interferon- $\beta$ and early dynamic changes of blood $\mathrm{T}$ cell clones have been observed in patients who received radiotherapy and demonstrated greater systemic immune responses (9), suggesting that radiotherapy can strongly activate antitumour immunity. Furthermore, DNA damage signalling after irradiation also stimulates innate immune responses via cyclic GMP-AMP synthase (cGAS)/stimulator of IFN genes (STING)- or the melanoma differentiation-associated gene 5/retinoic acid-inducible gene I (RIG-I)/mitochondrial antiviral-signalling protein pathways. These signals promote the release of interferons (10-13). Thus, although radiotherapy can be a promising tool to stimulate the antitumour immune response, positive stimulation may not fully achieve antitumour effects due to the undesired immune-suppressive signal, for example, PD-L1 up-regulation (14); especially if radiotherapy stimulates the immune-suppressive signal. Thus, it is important to understand the regulation of immune response in the context of its ligand expression in each patient to effectively implement this combination therapy.

To date, multiple pathways regarding the regulation of PD-L1 expression in tumours have been suggested. Microsatellite instability induced by defects in mismatch repair $(15,16)$ and $\mathrm{PD}-\mathrm{L} 1$ expression $(17)$ have been proposed as predictive markers for the efficacy of anti-PD-1/PD-L1 antibodies monotherapy. In the context of DNA damage-dependent regulation of PD-L1 expression, our previous study indicated that PD-L1 expression is up-regulated at the transcriptional level (14). This up-regulation was dependent on STAT1/3-IRF1 after the activation of ataxia telangiectasia mutated/ataxia telangiectasia and Rad3-related (ATR)/checkpoint kinase 1 (Chk1) (14). Our previous study also demonstrated in U2OS cells that PD-L1 up-regulation is enhanced in cells that are deficient in $\mathrm{Ku} 80$, a central regulator of non-homologous end joining (NHEJ).

The present study examined the correlation of mRNA expression between PD-L1 and NHEJ factors using The Cancer Genome Atlas (TCGA) dataset analysis. Ku 80 mRNA expression was negatively correlated with $P D-L 1$ mRNA expression in all the selected tissues. Moreover, the current study aimed to examine the relationship between Ku80 and PD-L1 expression in patients who received radiotherapy.

\section{Materials and methods}

TCGA analysis. Normalised RNA sequences of Ku80 and $P D-L 1$ expression status data provided by the TCGA project were downloaded from the Genomic Data Commons Data Portal (https://portal.gdc.cancer.gov). Datasets for colon adenocarcinoma (COAD), breast invasive carcinoma (BRCA), skin cutaneous melanoma (SKCM), lung adenocarcinoma (LUAD), head and neck squamous cell carcinoma (HNSC), uterine corpus endometrial carcinoma (UCEC) and cervical squamous cell carcinoma and endocervical adenocarcinoma (CESC) were analysed in the present study (18-24). Partial correlations and statistical significance presented as volcano plots were calculated using non-parametric Spearman's formula, shown as the coefficient rho (r) by GraphPad Prism v9.0 software (GraphPad Software, Inc.) and the XLSTAT 2021.1.1 add-in feature in Microsoft Excel v16.46 (Microsoft Corporation). $\mathrm{P}<0.05$ was considered to indicate a statistically significant difference. The total sample numbers for each dataset were 480 (COAD), 109 (BRCA), 470 (SKCM), 1,086 (LUAD), 502 (HNSC), 1,607 (UCEC) and 306 (CESC).

Patients and tumour characteristics. A total of 75 patients with cervical squamous cell carcinoma (median age, 62 years; range, 32-87 years) who met the following criteria were eligible for the present retrospective study: i) Pathologically-confirmed newly diagnosed cervical cancer by pathologists who were not associated with the present study; ii) treated with definitive platinum-based chemoradiotherapy (CRT group) or radiotherapy alone (RT group) at Gunma University Hospital between August 2009 and November 2013; iii) staged as IB1-IVA according to the FIGO classification 2008 (25); and iv) no previous exposure to radiotherapy or cytotoxic agents. Patients with recurrence were excluded from this study. Tumour samples from all patients before radiotherapy (pre-RT) and after 10 Gy of radiotherapy (10 Gy-RT) were used for pathological analysis and immunohistochemical staining. The time between the radiotherapy start date and the biopsy specimen date was 5-11 days. Most samples were collected on days 8-9 (89\% in the RT group and $95 \%$ in the CRT group). The time between the $10 \mathrm{~Gy}-\mathrm{RT}$ day and the specimen biopsy day ranged from 0-4 days. The majority of samples (75\%) were biopsied on the same day as that of receiving $10 \mathrm{~Gy}-\mathrm{RT}$. Patient characteristics were recorded according to tumour stage [stages IB, II, III and IV according to FIGO classification 2008 (25)] and age (Table I). The present study protocol was approved by the Institutional Review Board for clinical trials of Gunma University (Maebashi, Japan; approval no. HS2020-015). All patients provided their informed consent to participate in the study using the opt-out approach by public notice on the 
Table I. Characteristics of patients $(n=75)$ with cervical squamous cell carcinoma.

\begin{tabular}{lc}
\hline Characteristics & Value \\
\hline $\begin{array}{l}\text { Observation period, months } \\
\text { Median (range) }\end{array}$ & $63(8-120)$ \\
Age, years & \\
Median (range) & $62(32-87)$ \\
Treatment, n (\%) & \\
RT alone & $27(36 \%)$ \\
Concurrent CRT & $48(64 \%)$ \\
FIGO stage 2008, n (\%) & \\
IB & $11(15 \%)$ \\
II & $31(41 \%)$ \\
III & $31(41 \%)$ \\
IVA & $2(3 \%)$ \\
Lymph node metastasis in pelvis, n (\%) & \\
Positive & $36(48 \%)$ \\
Negative & $39(52 \%)$ \\
Para-aortic lymph node metastasis, n (\%) & \\
Positive & $6(8 \%)$ \\
Negative & $69(92 \%)$ \\
\hline
\end{tabular}

internet site of the Institutional Review Board for clinical trials of Gunma University.

Treatment of patients. All patients received definitive radiotherapy in combination with external body radiotherapy (EBRT) and intracavity brachytherapy (ICBT). EBRT typically used $10 \mathrm{MV}$ X-rays with 4-field irradiation. The most common EBRT dose and fractionation was 50 Gy in 25 fractions ( $2 \mathrm{~Gy} /$ fraction, once per day, five times weekly). EBRT was performed using a combination of total pelvic irradiation (20-40 Gy) followed by a central shielded irradiation at a $3-\mathrm{cm}$ width. The pelvic field was expanded to the metastatic area in patients with paraaortic lymph node metastases. Patients with lymph node metastases received an additional boost of 6-8 Gy in three to four fractions. ICBT was performed once a week with concurrent central shielding EBRT. EBRT was not performed on the day of ICBT administration. Three-dimensional image-guided brachytherapy was performed on all patients using a high-dose-rate ${ }^{192} \mathrm{Ir}$ remote after loading system (microSelectron ${ }^{\circledR}$ Digital; Elekta Instrument AB). The prescription dose for each ICBT was determined to cover $90 \%$ of the high-risk clinical target volume, using a total dose of $6 \mathrm{~Gy}$. Bulky and/or asymmetrical tumours were treated using additional interstitial irradiation. ICBT was typically performed four times. Concurrent chemotherapy was given to $64.0 \%$ (48/75) of the patients. According to the Japan Society of Gynecologic Oncology (JSGO) Guidelines 2017 for the Treatment of Uterine Cervical Cancer, cisplatin was usually administered weekly at a dose of $40 \mathrm{mg} / \mathrm{m}^{2}(26)$.

Immunohistochemical staining for PD-L1 and Ku80. PD-L1 expression in tumour cells and Ku80 expression in the nuclei of biopsy specimens excised from the cervical squamous cell carcinoma pre-RT and $10 \mathrm{~Gy}-\mathrm{RT}$ were evaluated using immunohistochemical staining. Biopsied samples were fixed in $10 \%$ buffered formalin for $24 \mathrm{~h}$ at room temperature and then dehydrated, degreased and paraffin-embedded. Paraffin embedded sections ( $4-\mu \mathrm{m}$ thick) were dewaxed in xylene at room temperature and rehydrated using a graded ethanol series. Endogenous peroxidase activity was blocked using a 10-min incubation in $0.3 \%$ hydrogen peroxide. After blocking by $10 \%$ goat normal serum (cat. no. 5425; Cell Signalling Technology, Inc.) for PD-L1 and Ku80 sections with a 20 min incubation at room temperature, the sections were incubated overnight with primary antibodies at $4^{\circ} \mathrm{C}$. The sections were incubated with a commercially available biotin-streptavidin immunoperoxidase kit [Histofine; SAB-PO (rabbit); cat. no. 424032; Nichirei Biosciences, Inc.; Nichirei Corporation] for $20 \mathrm{~min}$ at room temperature. Then, the sections were incubated for 5 min with diaminobenzidine at room temperature. The following antibodies were used: Monoclonal anti-PD-L1 antibody (1:100; clone E1L3N rabbit IgG; cat. no. 13684; Cell Signalling Technology, Inc.) and monoclonal anti-Ku80 antibody (1:200; clone C48E7, and rabbit IgG; cat. no. 2180T; Cell Signalling Technology, Inc.). The quality of the tumour samples was carefully evaluated and validated independently by two pathologists who are co-authors of the present study.

Quantification of PD-L1 and Ku80 in immunohistochemical images. All immunostaining images were obtained using the light microscope Leica DM4000 B (Leica Microsystems, $\mathrm{GmbH}$ ) equipped with a $\mathrm{x} 20$ objective lens. Expression of cytosolic PD-L1 was measured using public domain software ImageJ v1.53a (National Institutes of Health) as follows. In 32-bit colour images, which are commonly used in computers, each pixel has a red, blue and green signal intensity from 0 to 255 . Brown, which is composed of a higher red than blue signal, represents PD-L1 labelled by antibody in the immunohistochemical image. The nucleus is shown in blue and the background is shown in white, which consists of high red, blue and green signals. Therefore, brown is the only colour that has much higher red compared with blue signal in the microscopy images. The captured images were split into red, blue and green channels. The PD-L1 signal was enhanced by subtracting the blue signal from the red signal, taking advantage of the fact that all the subtracted values are returned as zero when the subtracted value is zero or less. The mean of the signal intensities in three areas of the tumour tissues were quantified. PD-L1 change was calculated as PD-L1 intensity (10 Gy-RT) subtracted by PD-L1 intensity (pre-RT). Furthermore, the percentages of tumour cells with cell-surface staining for PD-L1 were recorded and expressed as a tumour proportion score (TPS). If the TPS was $1-50 \%$, the sample was classified as PD-L1 1+, whereas samples with TPS $>50 \%$ were classified as PD-L1 2+. The population of Ku80-positive cells were measured using an opened source software QuPath (v0.1.2; Queen's University Belfast; Northern Ireland). The tumour tissue area was targeted to detect Ku80-positive cells and the Ku80 positivity was quantified.

Cell culture, small interfering (si)RNA-knockdown, flow cytometry and immunoblotting. HeLa cells were obtained 
from the American Type Culture Collection and cultured in Eagle's Minimum Essential Medium (FUJIFILM Wako Pure Chemical Corporation) with $10 \%$ foetal calf serum (Sigma Aldrich; Merck KGaA) at $37^{\circ} \mathrm{C}$. siRNA transfection was performed using DharmaFECT (GE Healthcare Dharmacon, Inc.). siRNA was added to suspended HeLa cells after trypsinisation (the final concentration of siRNA is $16 \mathrm{nM})$. Non-targeting siRNA was used as negative control. The siRNA oligonucleotides used are listed in Table SI. Cells were incubated after transfection for $48 \mathrm{~h}$ at $37^{\circ} \mathrm{C}$ before 10 Gy of irradiation. X-ray irradiation was performed using an MX-160Labo irradiator $(160 \mathrm{kVp} ; 1.06 \mathrm{~Gy} / \mathrm{min}$; mediXtec Corporation).

For immunoblotting, cells were harvested at $48 \mathrm{~h}$ after transfection with $\mathrm{x} 1$ Sample Buffer [50-mM Tris, 2\% sodium dodecyl sulphate, $6 \%$ glycerol, $1 \%$ (w/w) 3-mercapto1,2-propanediol and $0.008 \%$ bromophenol blue] following PBS wash. Harvested samples were boiled at $95^{\circ} \mathrm{C}$ for $5 \mathrm{~min}$ and sonicated using a Q55 Sonicator Ultrasonic Homogeniser (QSonica LLC; power amplification $15 \%$ for $5 \mathrm{sec}$, twice) (27). The number of cells each condition was confirmed to align the protein amount as the previous study. Subsequently, samples (10 $\mu \mathrm{l} /$ lane) were loaded onto 4-20\% Mini-PROTEAN TGX Precast Protein gels (Bio-Rad Laboratories, Inc.) and were run in Mini-PROTEAN Tetra Vertical Electrophoresis Cell (Bio-Rad Laboratories, Inc.) at $200 \mathrm{~V}$ for $30 \mathrm{~min}$. They were then transferred onto a BioTrace NT Nitrocellulose Transfer Membrane (Pall Life Sciences) using a Mini-PROTEAN Tetra Vertical Electrophoresis Cell (Bio-Rad Laboratories, Inc.) at $100 \mathrm{~V}$ for $1 \mathrm{~h}$. The membrane was incubated overnight at room temperature with anti-Ku80 (Rabbit mAb; 1:2,000; cat. no. 2180; Cell Signaling Technology, Inc.) and anti-Actin antibodies (mouse mAb, 1:5,000; cat. no. 3700; Cell Signaling Technology, Inc.), which served as the primary antibodies. After the membranes were washed trice with $\mathrm{x} 1$ Tris-buffered saline (TBST; $0.05 \%$ Tween 20), they were incubated with goat anti-rabbit $\operatorname{IgG}$, horseradish peroxidase-conjugated antibodies (1:4,000; cat. no. 7074; Cell Signalling Technology, Inc.) or anti-mouse $\mathrm{IgG}$, horse anti-mouse horseradish peroxidase-conjugated antibodies (1:4,000; cat. no. 7076; Cell Signalling Technology, Inc.) at room temperature for $1 \mathrm{~h}$. After being washed trice with $\mathrm{x} 1 \mathrm{TBST}$, the membrane was reacted with ECL Western Blotting Detection Reagent (GE Healthcare), and chemical luminescence was detected using a LAS-600 Bioimaging Analyser System (Azure Biosystems, Inc.). The signal intensities of Ku80 and Actin were measured using ImageJ. The signal intensity of Ku80 was normalized to that of Actin; subsequently, the Ku80 signal intensity ratio was normalized to the Ku80 signal intensity in HeLa cells transfected with siControl.

Cells were exposed to 10 Gy of irradiation then incubated for $48 \mathrm{~h}$ prior to flow cytometry analysis. Harvested cells were washed and collected with ice-cold $1 \mathrm{mM}$ EDTA-phosphate-buffered saline and then stained with anti-PD-L1 antibodies (clone E1L3N rabbit IgG; cat. no. 13684; Cell Signalling Technology, Inc.) for $20 \mathrm{~min}$ on ice. The fluorochrome used was APC. Dead cells detected by propidium iodide (Sigma-Aldrich; Merck KGaA) were excluded from the analysis. Flow cytometry analysis was performed on an Attune NxT Flow Cytometer (Thermo Fisher Scientific, Inc.). Mean fluorescence intensity (MFI) of the PD-L1 was calculated as: MFI (PD-L1)-MFI (isotype control).

Statistical analysis. The correlation between Ku80 positivity and PD-L1 signal intensity was evaluated using Spearman's rank correlation coefficient. Clinical outcomes were calculated using the Kaplan-Meier method, and statistically significant differences were confirmed using the log-rank test. Mann-Whitney U test was used to compare Ku80 positivity in pre-RT samples and classification of PD-L1 expression. MFI in flow cytometry analysis was compared using unpaired Student's two-tailed t-test. Results were shown as mean \pm error bars, which represent the standard error of three samples in the experiment. $\mathrm{P}<0.05$ was considered to indicate a statistically significant difference. All statistical analyses were performed using SPSS (v24; IBM Corp.).

\section{Results}

TCGA analysis demonstrated negative correlation between $K u 80$ and PD-L1. TCGA datasets were used in the present study to investigate the relationship of gene expression between PD-L1 and DNA double-strand break (DSB) repair factors in tumour cells. Our previous study reported that tumour specimens harbouring mutations in NHEJ genes (Ku70/80) exhibit greater PD-L1 expression after irradiation in several cancer types (14). To determine whether the mRNA expression of NHEJ factors demonstrated a correlation with $P D-L 1$ mRNA expression, TCGA analyses between central NHEJ factors $[K u 80, K u 70$, DNA-dependent protein kinase catalytic subunit (DNA-PKcs), X-ray repair cross-complementing protein 4 (XRCC4) and DNA ligase 4] and $P D-L 1$ were performed (Table II). Fig. 1 presents the results of the seven cancer tissues, COAD, BRCA, SKCM, LUAD, HNSC, UCEC and CESC. Spearman's rho was $<0$ for all cancer types in $K u 80$ (Fig. 1; Table II). Because the high proliferative conditions in cancer cells can induce an unrepairable amount of DNA replication-associated genotoxic stress, including DSBs (28), it is hypothesized that the result of TCGA analysis reflects the situation in cells that are exposed to exogenous DNA damage, such as ionising radiation. Therefore, the present study investigated the correlation between Ku80 and PD-L1 in clinical specimens that received radiotherapy.

PD-L1 expression is negatively correlated with Ku80 expression in cervical squamous cell carcinoma samples treated with radiotherapy. Our previous study reported that PD-L1 expression is up-regulated in cervical squamous cell carcinoma specimens after being treated with $10 \mathrm{~Gy}$ of radiotherapy (29). The present study investigated whether Ku80 expression was correlated with PD-L1 expression in the clinical sample sets with or without radiotherapy. Ku80 and PD-L1 expression levels were quantitatively measured in immunohistochemical stained specimens using ImageJ and QuPath (Figs. S1 and S2). Although there was no obvious correlation between Ku80 and PD-L1 in pre-RT specimens [Spearman's rank correlation coefficient $(\mathrm{SRCC})=0.167$, $\mathrm{P}=0.152$; Fig. 2A], PD-L1 in 10 Gy-RT exhibited a weak negative correlation with Ku80 positivity in pre-RT specimens (SRCC $=-0.274, \mathrm{P}=0.017$; Fig. 2A). Next, to investigate the 
Table II. TCGA analysis.

\begin{tabular}{|c|c|c|c|c|c|}
\hline Gene & Study & Correlation & Approximation formula & Spearman's rho (r) & P-value \\
\hline \multirow[t]{7}{*}{ Ки 80} & COAD & Negative & $y=-0.001536 x+32430$ & -0.079 & 0.070 \\
\hline & BRCA & Negative & $y=-0.003198 x+34244$ & -0.005 & 0.869 \\
\hline & SKCM & Negative & $y=-0.01340 x+62900$ & -0.157 & 0.360 \\
\hline & LUAD & Negative & $y=-0.04393 x+181872$ & -0.236 & $8.88 \times 10^{-16}$ \\
\hline & HNSC & Negative & $y=-0.01328 x+136161$ & -0.053 & 0.232 \\
\hline & UCEC & Negative & $y=-0.009837 x+30808$ & -0.397 & $2.21 \times 10^{-5}$ \\
\hline & CESC & Negative & $y=-0.0433 x+177708$ & -0.151 & 0.008 \\
\hline \multirow[t]{7}{*}{ Ku 70} & COAD & Negative & $y=-0.003319 x+38104$ & $4.81 \times 10^{-4}$ & 0.991 \\
\hline & BRCA & Positive & $y=0.0009455 x+26915$ & -0.025 & 0.374 \\
\hline & SKCM & Negative & $y=-0.01050 x+71586$ & -0.229 & 0.179 \\
\hline & LUAD & Positive & $y=0.001316 x+118475$ & -0.154 & $1.87 \times 10^{-7}$ \\
\hline & HNSC & Negative & $y=-0.01467 x+165072$ & -0.108 & 0.016 \\
\hline & UCEC & Positive & $\mathrm{y}=0.001272 \mathrm{x}+10171$ & -0.090 & 0.353 \\
\hline & CESC & Negative & $y=-0.008341 x+141397$ & -0.110 & 0.054 \\
\hline \multirow[t]{7}{*}{$D N A-P K c s$} & COAD & Positive & $\mathrm{y}=0.01038 \mathrm{x}+25588$ & -0.108 & 0.014 \\
\hline & BRCA & Positive & $y=0.003123 x+27447$ & 0.047 & 0.098 \\
\hline & SKCM & Negative & $\mathrm{y}=-0.01111 \mathrm{x}+45835$ & -0.171 & 0.320 \\
\hline & LUAD & Positive & $\mathrm{y}=0.01127 \mathrm{x}+117308$ & -0.069 & 0.019 \\
\hline & HNSC & Negative & $y=-0.03491 x+137903$ & -0.035 & 0.432 \\
\hline & UCEC & Negative & $y=-0.008411 x+16815$ & -0.371 & $7.98 \times 10^{-5}$ \\
\hline & CESC & Negative & $y=-0.1111 x+163343$ & -0.074 & 0.198 \\
\hline \multirow[t]{7}{*}{$X R C C 4$} & COAD & Positive & $y=0.1244 x+19506$ & 0.069 & 0.116 \\
\hline & BRCA & Positive & $y=0.03546 x+26440$ & 0.077 & 0.007 \\
\hline & SKCM & Positive & $y=0.5896 x+763.2$ & 0.280 & 0.098 \\
\hline & LUAD & Positive & $y=0.1271 x+111566$ & 0.062 & 0.036 \\
\hline & HNSC & Positive & $y=0.3040 x+87953$ & 0.175 & $8.04 \times 10^{-5}$ \\
\hline & UCEC & Negative & $y=-0.02123 x+14814$ & -0.233 & 0.014 \\
\hline & CESC & Negative & $y=-0.1882 x+137753$ & 0.142 & 0.012 \\
\hline \multirow[t]{7}{*}{ LIG4 } & COAD & Positive & $y=0.07266 x+25129$ & 0.022 & 0.615 \\
\hline & BRCA & Positive & $\mathrm{y}=0.08249 \mathrm{x}+23007$ & 0.078 & 0.006 \\
\hline & SKCM & Positive & $y=0.08564 x+33814$ & 0.102 & 0.552 \\
\hline & LUAD & Positive & $y=0.1574 x+109242$ & 0.099 & 0.001 \\
\hline & HNSC & Positive & $y=0.08293 x+110697$ & 0.097 & 0.029 \\
\hline & UCEC & Negative & $\mathrm{y}=-0.02302 \mathrm{x}+14377$ & -0.009 & 0.924 \\
\hline & CESC & Negative & $y=-0.07419 x+122540$ & -0.029 & 0.605 \\
\hline
\end{tabular}

COAD, colon adenocarcinoma; BRCA, breast invasive carcinoma; SKCM, skin cutaneous melanoma; LUAD, lung adenocarcinoma; HNSC, head and neck squamous cell carcinoma; UCEC, uterine corpus endometrial carcinoma; CESC, cervical squamous cell carcinoma and endocervical adenocarcinoma; DNA-PKcs, DNA-dependent protein kinase, catalytic subunit; XRCC4, X-ray repair cross-complementing protein 4; LIG4, DNA ligase 4.

impact of radiotherapy on PD-L1 expression in the context of Ku80 positivity, PD-L1 changes in 10 Gy-RT were plotted against Ku80 positivity of pre-RT specimens. Notably, a significant negative correlation between Ku80 positivity of pre-RT and PD-L1 changes was revealed ( $\mathrm{SRCC}=-0.379$; $\mathrm{P}<0.001$; Fig. 2B). Representative images are shown in Fig. 3. To validate the computational quantification analysis, the same analysis scoring was performed using a manual classification (TPS) in the same specimens. Similar to the results presented in Fig. 2B, a negative correlation was observed between Ku80 positivity of pre-RT and PD-L1 changes after
10 Gy-RT (Fig. S3). In addition, the potential association between Ku80 positivity and clinical outcomes was examined. Overall survival, progression-free survival and local control did not exhibit significant differences with Ku80 expression (Fig. S4). These data suggested that PD-L1 expression after 10 Gy-RT was negatively correlated with Ku80, whereas there was no correlation between PD-L1 expression and patients' outcome.

PD-L1 up-regulation after irradiation is enhanced in Ku80-depleted HeLa cells. To confirm radiation-induced 

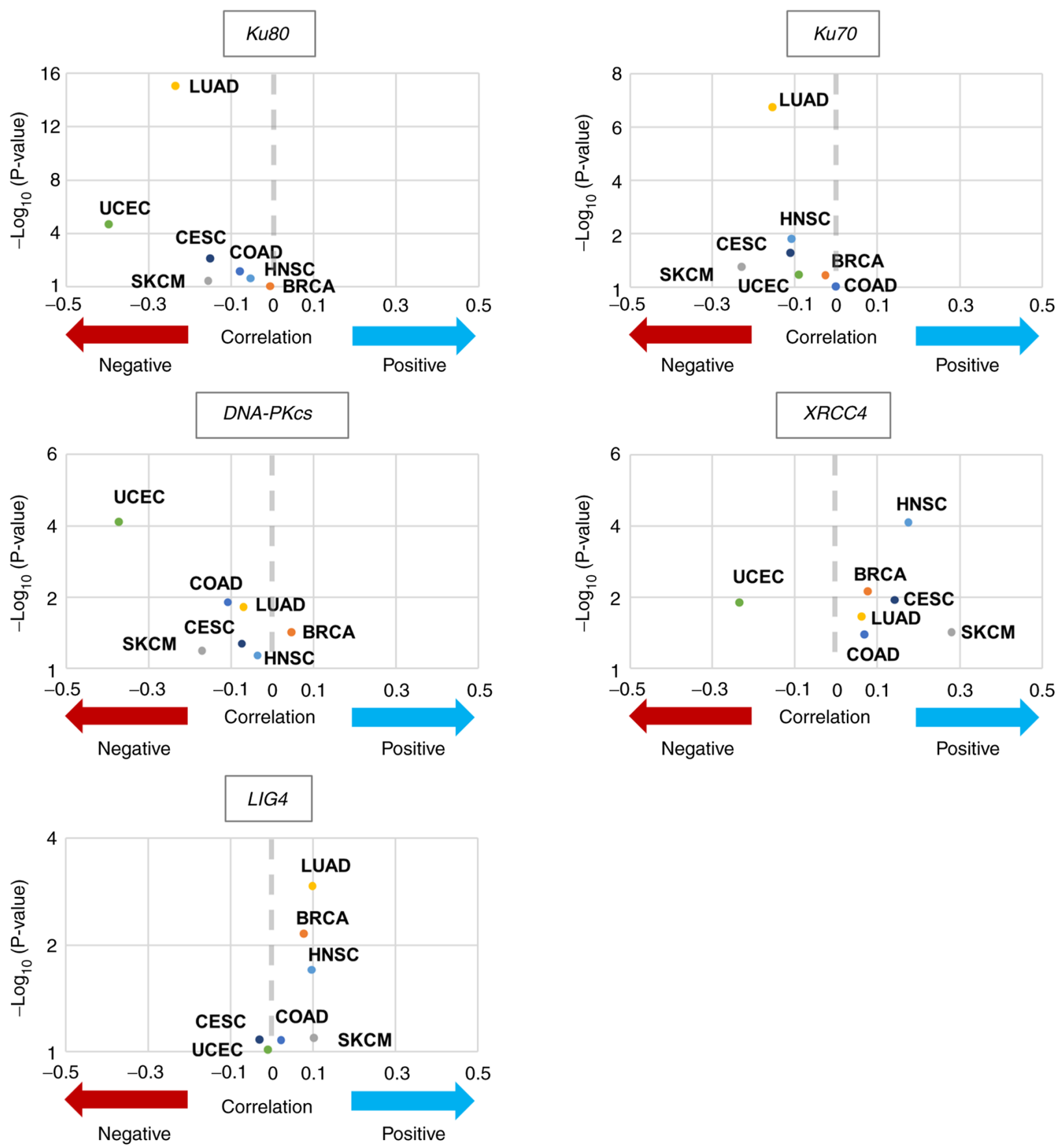

Figure 1. Correlation between PD-L1 gene expression and DNA double-strand break repair factors. Volcano plots indicating the correlation between PD-L1 expression and central non-homologous end joining factors expression levels. Mutation statuses were provided by The Cancer Genome Atlas project were downloaded from the Genomic Data Commons Data Portal. BRCA, breast invasive carcinoma; CESC, cervical squamous cell carcinoma and endocervical adenocarcinoma; COAD, colon adenocarcinoma; HNSC, head and neck squamous cell carcinoma; LUAD, lung adenocarcinoma; SKCM, skin cutaneous melanoma; UCEC, uterine corpus endometrial carcinoma; DNA-PKcs, DNA-dependent protein kinase, catalytic subunit; XRCC4, X-ray repair cross-complementing protein 4; LIG4, DNA ligase 4.

PD-L1 up-regulation in a Ku80-depleted cervical cancer cell line in vitro, flow cytometry analysis was performed in HeLa cells transfected with Ku80 siRNA to examine the levels of cell-surface PD-L1 expression after X-ray irradiation. Consistent with the immunohistochemistry results seen in the tumour specimens, PD-L1 up-regulation induced by $10 \mathrm{~Gy}$ $\mathrm{X}$-ray irradiation was significantly increased in Ku80-depleted cells compared with controls treated with X-ray irradiation (Figs. 4, S5 and S6), suggesting that cell-surface PD-L1 expression was enhanced in Ku80-depleted condition.

\section{Discussion}

The present study revealed that $K u 80$ mRNA expression in tumours was negatively correlated with $P D-L 1$ expression using TCGA database analysis. Notably, in the clinical sample sets from the present study, PD-L1 induction after $10 \mathrm{~Gy}-\mathrm{RT}$ was inversely correlated with Ku80 expression in pre-RT specimens. In addition, significant up-regulation of PD-L1 expression after irradiation was confirmed in Ku80-depleted HeLa cells compared with irradiated controls, supporting 

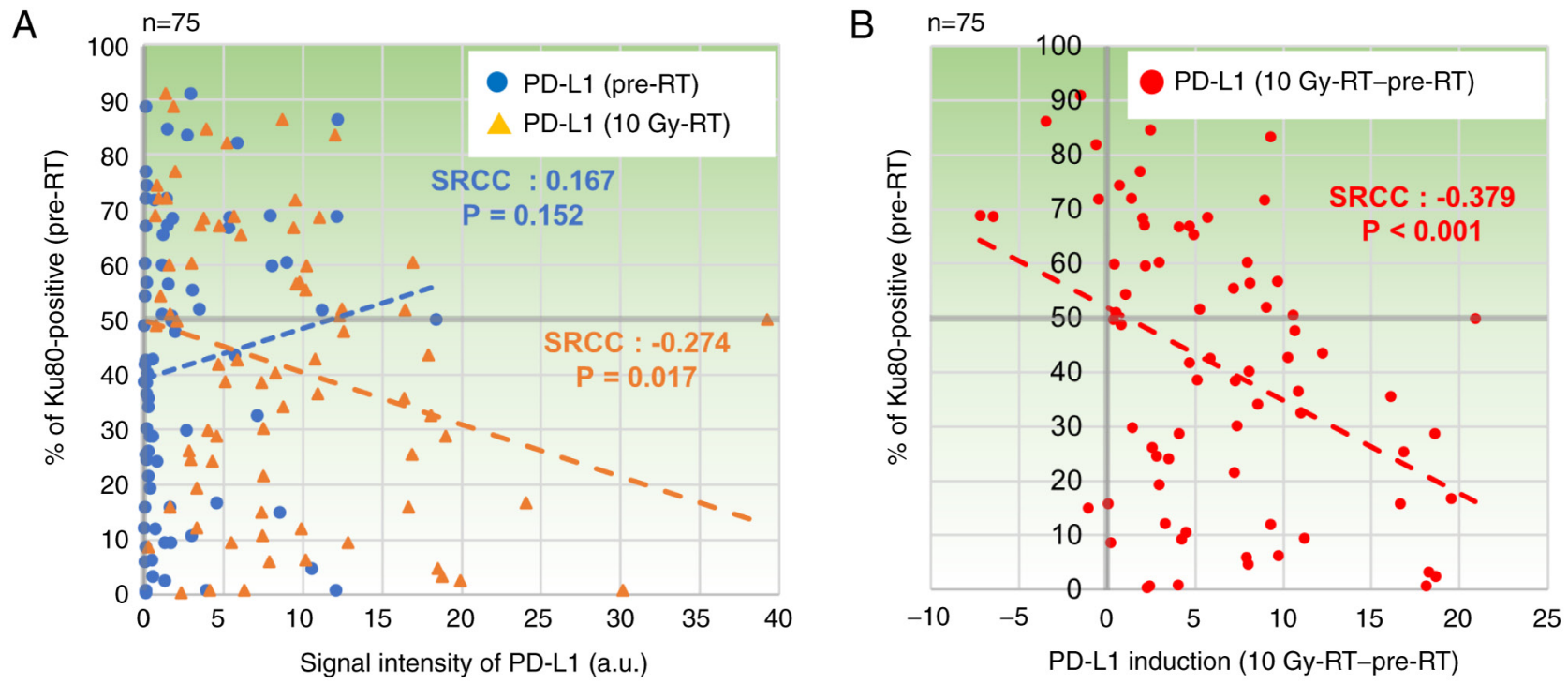

Figure 2. Correlations between PD-L1 expression and Ku80 positivity in cervical squamous cell carcinoma after treatment with radiotherapy. (A) Scatter plot showing the relationship between PD-L1 expression (pre-RT and 10 Gy-RT) and Ku80 positivity using ImageJ and QuPath, respectively. (B) Scatter plot showing the relationship between PD-L1 changes comparing pre-RT and 10 Gy-RT and Ku80 positivity. SRCC, Spearman's rank correlation coefficient; PD-L1, programmed cell death-1 ligands; RT, radiotherapy.
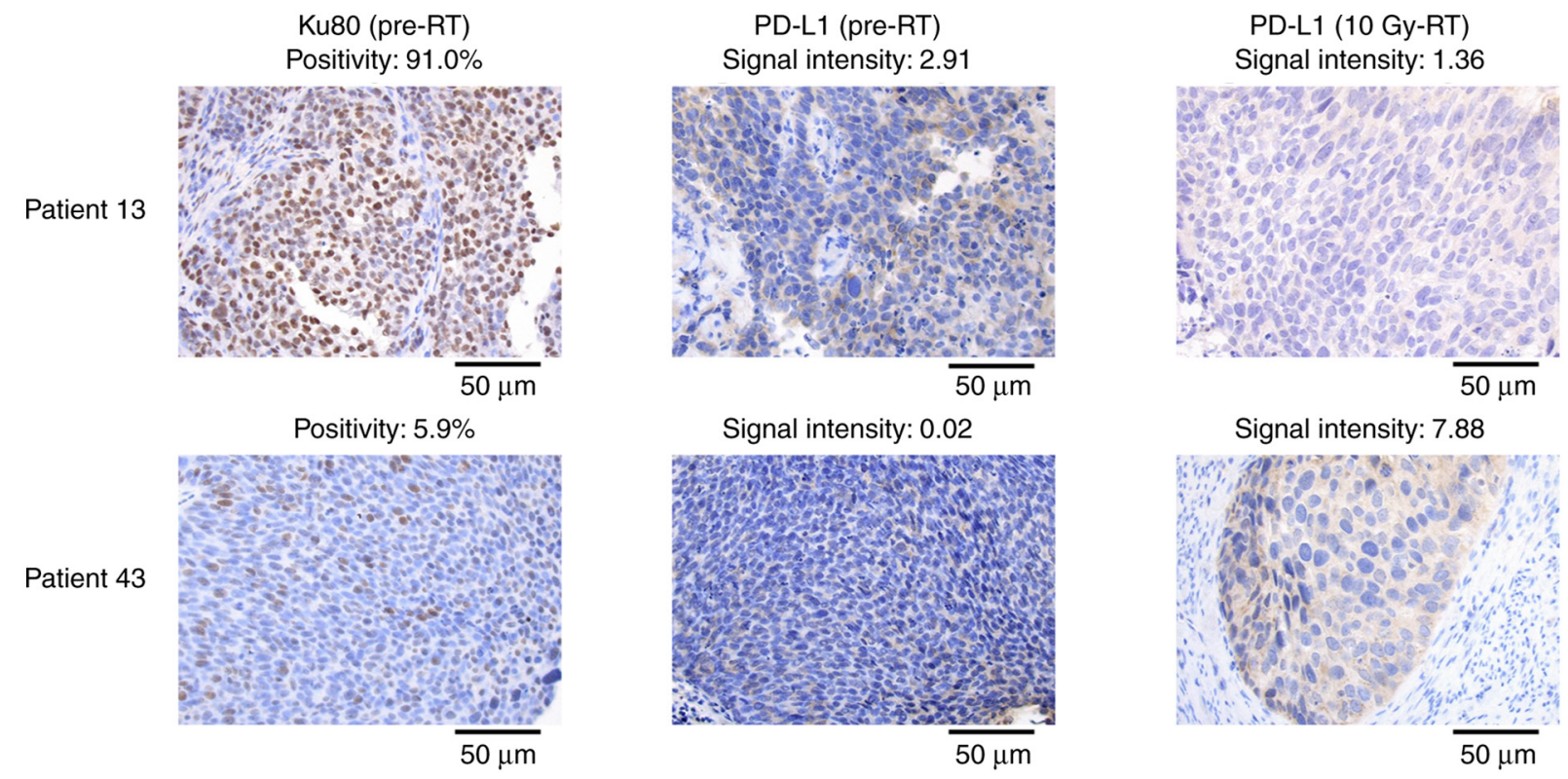

Figure 3. Representative images of immunohistochemical staining. The upper three images show specimens from the same patient with $91.0 \%$ Ku80 positivity (relatively high) and low PD-L1 induction following 10 Gy-RT. The lower three images show specimens from another patient with $5.9 \%$ Ku 80 positivity (relatively low) and high PD-L1 induction following 10 Gy-RT. Scale bar, $50 \mu \mathrm{m}$. PD-L1, programmed cell death-1 ligands; RT, radiotherapy.

supporting the hypothesis that low Ku80 expression can affect PD-L1 up-regulation after radiotherapy.

Our previous studies reported that DNA damage signalling induced by irradiation or oxidative damage up-regulates PD-L1 expression at the transcriptional level $(14,30)$. The increase in $P D$ - $L 1$ mRNA transcription consequently leads to enhanced PD-L1 presentation on the cell surface. This up-regulation is dependent on ATR/Chk1 (14). Since ATR/Chk1 activation is associated with the progression of DSB end resection (high resection causes greater ATR/Chk1 signalling), the magnitude of resection is correlated with PD-L1 expression $(31,32)$. Consistent with this, our previous study revealed that $\mathrm{Ku} 80$ depletion, which causes greater DSB end resection, leads to greater ATR/Chk1 signalling and PD-L1 expression in cancer cell lines such as U2OS cells (14).

The present study revealed that Ku80-depleted HeLa cells also expressed greater PD-L1 after irradiation. Together with the findings of TCGA analysis, which demonstrated that low $K u 80$ mRNA expression was correlated with high $P D-L 1$ expression in all the cancer types analysed in this study (colon adenocarcinoma, breast invasive carcinoma, skin cutaneous melanoma, lung adenocarcinoma, head and neck squamous cell carcinoma, uterine corpus endometrial carcinoma, cervical squamous cell carcinoma and endocervical adenocarcinoma), 
A
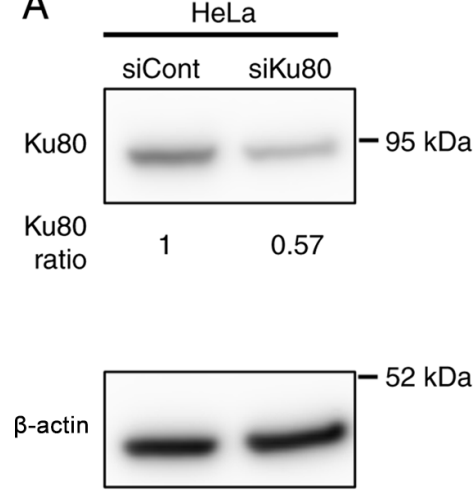

B

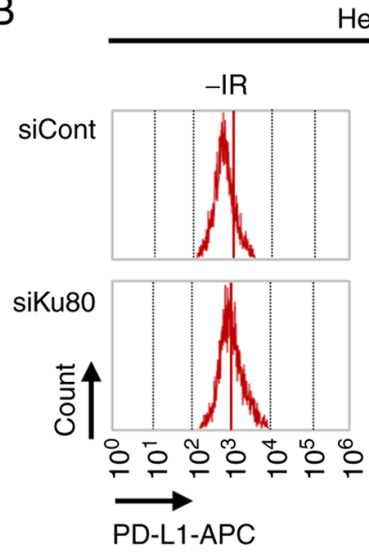

HeLa

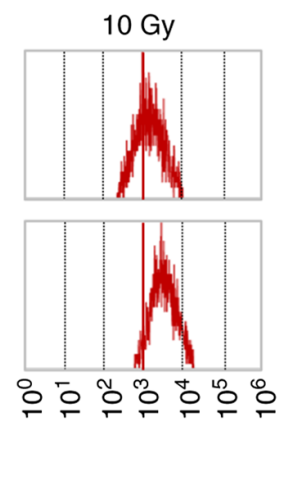

C

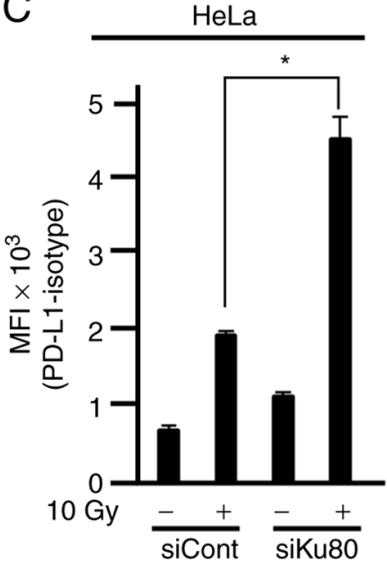

Figure 4. PD-L1 expression is enhanced after irradiation of Ku80-depleted HeLa cells. (A) Immunoblotting of Ku80 in HeLa cells transfected with scrambled siCont RNA or siKu80. (B) Flow cytometry histogram of PD-L1 expression after 10 Gy irradiation of HeLa cells. Cell-surface PD-L1 expression was examined at $48 \mathrm{~h}$ after $10 \mathrm{~Gy}$ irradiation. (C) Depletion of Ku80 enhanced the up-regulation of cell-surface PD-L1 expression after irradiation. Error bars represent the standard error of three samples in the experiment. ${ }^{*} \mathrm{P}<0.05$. si, short interfering; cont, control; PD-L1, programmed cell death-1 ligands; IR, irradiation; MFI, mean fluorescence intensity.

Ku80 expression levels were associated with PD-L1 expression, particularly when cells were exposed to genotoxic stress. The results also suggested that greater PD-L1 up-regulation can occur in patients who have received radiotherapy if $\mathrm{Ku} 80$ expression is low.

To date, multiple pathways regarding PD-L1 expression after DNA damage have been suggested, such as cytosolic sensors cGAS/RIG-I transducing immune-activating signal releasing interferon stimulate genes following the recognition of cytosolic DNA/RNA released from the nucleus (10-12). Since radiotherapy can generate micronuclei that should contain DNA/RNA (33), further studies are required to examine the involvement of this pathway.

The present study quantified the signal intensity of PD-L1 using software. Although the signals from inside the cell or the cell surface were unable to be differentiated, which seems to be important to detect functional PD-L1 on cell surface, the software approach has advantages in measuring an interval scale of their expression. PD-L1 expression was also examined using a manual classification method scored by cell-surface staining for PD-L1. Notably, the present study obtained similar results using both methods. Thus, it was hypothesized that this computational measurement could be a useful tool to quantify PD-L1 expression; however, it must be carefully accessed depending on the type of targets.

Limitations of the present study included the limited number of clinical samples. As there are multiple regulators of PD-L1 expression (34), other regulators may also affect the PD-L1 expression after RT in clinical samples. Data variability may be minimised by analysing the expression of PD-L1 in subgroups classified by regulators of PD-L1 expression other than Ku80. Future studies involving larger cohorts may help in further elucidating the relationship between PD-L1 and Ku80 expression in clinical settings. In addition, clinical trials are required to analyse whether the increase in PD-L1 after RT in clinical tumours with low Ku80 expression affects ICI treatment in the future. Although overexpression of Ku80 was not examined in the present study, it should be examined in future studies to determine the magnitude of Ku80 expression; for example, low Ku80 expression compared with normal levels was associated with PD-L1 up-regulation after X-ray irradiation as indicated in the present study.

Recent radiotherapy technologies are highly developed and enable radiation oncologists to target cancer using high-precision radiotherapy, while also avoiding irradiation to the surrounding normal tissue. These technological developments increase the importance of controlling tumour cells outside the irradiation field for long-term survival of patients (35). For several decades, radiation has been considered a potent inducer of immune activation (36); however, the clinical outcomes of patients treated with combination therapy differ per case, and the mechanisms underlying this difference need to be investigated. Therefore, the radiotherapy-dependent immune stimuli are still unknown.

Notably, the situation of radiotherapy, such as dose fractionation and radiation quality, affects immune responses. For example, hypofractionated radiation at 8-12 Gy per fraction activates the cGAS/STING pathway more effectively compared with a higher single dose of $\geq 20$ Gy (11). Also, our recent study reported that PD-L1 up-regulation is more significantly induced by high linear energy transfer carbon-ion irradiation compared with X-rays (27). Hence, the reaction of the immune system after radiotherapy is comprehensive. To identify the optimal modality for radiation/ICI combination therapy, further research regarding optimal dose/fractionation, field settings, combination timing and type of irradiation are required. By contrast, recent studies have demonstrated that radiotherapy also up-regulates PD-L1 in tumours (34,37-39); therefore, combination therapy with ICIs is promising. Indeed, phase III clinical trials have revealed that combination therapy improves clinical outcomes in patients with locally advanced non-small cell lung cancer or metastatic castration-resistant prostate cancer (40-43). However, this may not be applicable to all patients with cancer from the viewpoint of precision medicine; for example, PD-L1 is not effectively up-regulated in some patients with certain genetic backgrounds, such as those without Ku80 mutation, as indicated in the present study. 
The present study demonstrated that PD-L1 expression is induced by radiotherapy and can be affected by the expression levels of Ku80 prior to radiotherapy. The present study did not reveal any differences in Ku80 positivity with patient outcomes, which may be because of the multiple functions of Ku80 that are involved in both tumour immunity and DNA repair $(14,44)$. Furthermore, PD-L1 expression is not significantly correlated with clinical outcomes in the same cohort in our previous study (29). Although whether PD-L1 expression can be a predictive marker of ICIs is still debatable, the findings of the present study suggest that Ku80 may be a potential biomarker for radiation/ICI combination therapy. Further studies into this type of approach will provide important data to determine the best strategy for radiation/ICI combination therapy.

\section{Acknowledgements}

The authors thank Dr Yuya Yoshimoto (Fukushima Medical University, Fukushima; Gunma University, Maebashi, Japan) for his assistance in performing the immunohistochemical analysis. The authors thank Mr Koji Isoda (Gunma University, Maebashi, Japan) for his technical assistance in performing the immunohistochemical analysis.

\section{Funding}

This study was supported by JSPS KAKENHI (grant nos. JP 17H04713 and JP19K08195), the Takeda Science Foundation, the Uehara Memorial Foundation, the Astellas Foundation for Research on Metabolic Disorders, The Kanae Foundation for the Promotion of Medical Science, the Yasuda Memorial Medicine Foundation and the Nakajima Foundation. This study was also supported by the Program of the Network-Type Joint Usage/Research Centre for Radiation Disaster Medical Science of Hiroshima University, Nagasaki University and Fukushima Medical University and the Grants-in-Aid from the Ministry of Education, Culture, Sports, Science and Technology of Japan for programmes for Leading Graduate Schools, Cultivating Global Leaders in Heavy Ion Therapeutics and Engineering.

\section{Availability of data and materials}

The datasets used and/or analyzed during the current study are available from the corresponding author on reasonable request.

\section{Authors' contributions}

TK, YM, HS, TBMP, YU, KO, SK, KS, HI, HY and AS performed the experiments and analysed the data. TK, TBMP, HS and AS wrote the manuscript. TK, YM, SN and KO coordinated the clinics, carried out the treatment and participated in the patient follow-up. SG, TN and TO contributed conception and design, and reagents/materials/analysis tools and gave final approval for the manuscript version to be published. TK and HS confirm the authenticity of all the raw data. HS and TO supervised the study with the support from AS. All authors have read and approved the final manuscript.

\section{Ethics approval and consent to participate}

The present study was approved by the Institutional Review Board for clinical trials of Gunma University (approval no. HS2020-015; Maebashi, Japan). The protocol is described on the hospital website, and subjects were provided the opportunity to opt-out; therefore, no additional consent was required from patients.

\section{Patient consent for publication}

Not applicable.

\section{Competing interests}

The authors declare that they have no competing interests.

\section{References}

1. Sharma P and Allison JP: Immune checkpoint targeting in cancer therapy: Toward combination strategies with curative potential. Cell 161: 205-214, 2015.

2. Galon J and Bruni D: Approaches to treat immune hot, altered and cold tumours with combination immunotherapies. Nat Rev Drug Discov 18: 197-218, 2019.

3. Yap TA, Parkes EE, Peng W, Moyers JT, Curran MA and Tawbi HA: Development of immunotherapy combination strategies in cancer. Cancer Discov 11: 1368-1397, 2021.

4. Guillem JG, Chessin DB, Cohen AM, Shia J, Mazumdar M, Enker W, Paty PB, Weiser MR, Klimstra D, Saltz L, et al: Long-term oncologic outcome following preoperative combined modality therapy and total mesorectal excision of locally advanced rectal cancer. Ann Surg 241: 829-836; discussion 836-838, 2005.

5. Golden EB, Frances D, Pellicciotta I, Demaria S, Helen Barcellos-Hoff M and Formenti SC: Radiation fosters dose-dependent and chemotherapy-induced immunogenic cell death. Oncoimmunology 3: e28518, 2014.

6. Diamond JM, Vanpouille-Box C, Spada S, Rudqvist NP, Chapman JR, Ueberheide BM, Pilones KA, Sarfraz Y, Formenti SC and Demaria S: Exosomes Shuttle TREX1-Sensitive IFN-Stimulatory dsDNA from irradiated cancer cells to DCs. Cancer Immunol Res 6: 910-920, 2018.

7. Rodriguez-Ruiz ME, Vanpouille-Box C, Melero I, Formenti SC and Demaria S: Immunological mechanisms responsible for Radiation-Induced Abscopal effect. Trends Immunol 39: 644-655, 2018.

8. Reits EA, Hodge JW, Herberts CA, Groothuis TA, Chakraborty M, Wansley EK, Camphausen K, Luiten RM, de Ru AH, Neijssen J, et al: Radiation modulates the peptide repertoire, enhances MHC class I expression, and induces successful antitumor immunotherapy. J Exp Med 203: 1259-1271, 2006.

9. Formenti SC, Rudqvist NP, Golden E, Cooper B, Wennerberg E, Lhuillier C, Vanpouille-Box C, Friedman K, Ferrari de Andrade L, Wucherpfennig KW, et al: Radiotherapy induces responses of lung cancer to CTLA-4 blockade. Nat Med 24: 1845-1851, 2018.

10. Harding SM, Benci JL, Irianto J, Discher DE, Minn AJ and Greenberg RA: Mitotic progression following DNA damage enables pattern recognition within micronuclei. Nature 548: 466-470, 2017.

11. Vanpouille-Box C, Alard A, Aryankalayil MJ, Sarfraz Y, Diamond JM, Schneider RJ, Inghirami G, Coleman CN, Formenti SC and Demaria S: DNA exonuclease Trex1 regulates radiotherapy-induced tumour immunogenicity. Nat Commun 8: 15618, 2017.

12. Yamazaki T,Kirchmair A,Sato A,Buqué A, Rybstein M,PetroniG, Bloy N, Finotello F, Stafford L, Navarro Manzano E, et al: Mitochondrial DNA drives abscopal responses to radiation that are inhibited by autophagy. Nat Immunol 21: 1160-1171, 2020.

13. Feng $X$, Tubbs A, Zhang C, Tang M, Sridharan S, Wang C, Jiang D, Su D, Zhang H, Chen Z, et al: ATR inhibition potentiates ionizing radiation-induced interferon response via cytosolic nucleic acid-sensing pathways. EMBO J 39: e104036, 2020. 
14. Sato H, Niimi A, Yasuhara T, Permata TBM, Hagiwara Y, Isono M, Nuryadi E, Sekine R, Oike T, Kakoti S, et al: DNA double-strand break repair pathway regulates PD-L1 expression in cancer cells. Nat Commun 8: 1751, 2017.

15. Le DT, Durham JN, Smith KN, Wang H, Bartlett BR, Aulakh LK, Lu S, Kemberling H, Wilt C, Luber BS, et al: Mismatch repair deficiency predicts response of solid tumors to PD-1 blockade. Science 357: 409-413, 2017.

16. Marabelle A, Le DT, Ascierto PA, Di Giacomo AM, De Jesus-Acosta A, Delord JP, Geva R, Gottfried M, Penel N, Hansen AR, et al: Efficacy of Pembrolizumab in patients with noncolorectal high Microsatellite Instability/Mismatch Repair-Deficientcancer:Results from thePhaseIIKEYNOTE-158 Study. J Clin Oncol 38: 1-10, 2020.

17. Sunshine J and Taube JM: PD-1/PD-L1 inhibitors. Curr Opin Pharmacol 23: 32-38, 2015.

18. Cancer Genome Atlas Network: Comprehensive molecular characterization of human colon and rectal cancer. Nature 487: 330-337, 2012

19. Cancer Genome Atlas Network: Comprehensive molecular portraits of human breast tumours. Nature 490: 61-70, 2012.

20. Cancer Genome Atlas Network: Genomic classification of cutaneous melanoma. Cell 161: 1681-1696, 2015.

21. Cancer Genome Atlas Research Network: Comprehensive molecular profiling of lung adenocarcinoma. Nature 511: 543-550, 2014

22. Cancer Genome Atlas Network: Comprehensive genomic characterization of head and neck squamous cell carcinomas. Nature 517: 576-582, 2015.

23. Cancer Genome Atlas Research Network, Kandoth C, Schultz N Cherniack AD, Akbani R, Liu Y, Shen H, Robertson AG, Pashtan I, Shen R, et al: Integrated genomic characterization of endometrial carcinoma. Nature 497: 67-73, 2013.

24. Cancer Genome Atlas Research Network, Albert Einstein College of Medicine; Analytical Biological Services, Barretos Cancer Hospital, Baylor College of Medicine, Beckman Research Institute of City of Hope, Buck Institute for Research on Aging, Canada's Michael Smith Genome Sciences Centre, Harvard Medical School, Helen F. Graham Cancer Center \& Research Institute at Christiana Care Health Services, et al: Integrated genomic and molecular characterization of cervical cancer. Nature 543: 378-384, 2017.

25. Pecorelli S: Revised FIGO staging for carcinoma of the vulva, cervix, and endometrium. Int J Gynaecol Obstet 105: 103-104, 2009.

26. Ebina Y, Mikami M, Nagase S, Tabata T, Kaneuchi M, Tashiro H, Mandai M, Enomoto T, Kobayashi Y, Katabuchi H, et al: Japan Society of Gynecologic Oncology guidelines 2017 for the treatment of uterine cervical cancer. Int J Clin Oncol 24: 1-19, 2019.

27. Permata TBM, Sato H, Gu W, Kakoti S, Uchihara Y, Yoshimatsu Y, Sato I, Kato R, Yamauchi M, Suzuki K, et al: High linear energy transfer carbon-ion irradiation upregulates PD-L1 expression more significantly than X-rays in human osteosarcoma U2OS cells. J Radiat Res 62: 773-781, 2021.

28. Shrivastav M, De Haro LP and Nickoloff JA: Regulation of DNA double-strand break repair pathway choice. Cell Res 18: 134-147, 2008.

29. Mori Y, Sato H, Kumazawa T, Permata TBM, Yoshimoto Y, Murata K, Noda SE, Kaminuma T, Ando K, Oike T, et al: Analysis of radiotherapy-induced alteration of $\mathrm{CD}^{+} \mathrm{T}$ cells and PD-L1 expression in patients with uterine cervical squamous cell carcinoma. Oncol Lett 21: 446, 2021.

30. Permata TBM, Hagiwara Y, Sato H, Yasuhara T, Oike T, Gondhowiardjo S, Held KD, Nakano T and Shibata A: Base excision repair regulates PD-L1 expression in cancer cells. Oncogene 38: 4452-4466, 2019.

31. Jazayeri A, Falck J, Lukas C, Bartek J, Smith GC, Lukas J and Jackson SP: ATM- and cell cycle-dependent regulation of ATR in response to DNA double-strand breaks. Nat Cell Biol 8: 37-45, 2006
32. Rhind N: Changing of the guard: How ATM hands off DNA double-strand break signaling to ATR. Mol Cell 33: 672-674, 2009.

33. Kobayashi D, Oike T, Murata K, Irie D, Hirota Y, Sato H, Shibata A and Ohno T: Induction of micronuclei in cervical cancer treated with radiotherapy. J Pers Med 10: 110, 2020.

34. Sato H, Jeggo PA and Shibata A: Regulation of programmed death-ligand 1 expression in response to DNA damage in cancer cells: Implications for precision medicine. Cancer Sci 110: 3415-3423, 2019.

35. Chetty IJ, Martel MK, Jaffray DA, Benedict SH, Hahn SM, Berbeco R, Deye J, Jeraj R, Kavanagh B, Krishnan S, et al: Technology for innovation in radiation oncology. Int J Radiat Oncol Biol Phys 93: 485-492, 2015.

36. Lhuillier C, Rudqvist NP, Elemento O, Formenti SC and Demaria S: Radiation therapy and anti-tumor immunity: Exposing immunogenic mutations to the immune system. Genome Med 11: 40, 2019.

37. Dovedi SJ, Adlard AL, Lipowska-Bhalla G, McKenna C, Jones S, Cheadle EJ, Stratford IJ, Poon E, Morrow M, Stewart R, et al: Acquired resistance to fractionated radiotherapy can be overcome by concurrent PD-L1 blockade. Cancer Res 74: 5458-5468, 2014.

38. Patel KR, Martinez A, Stahl JM, Logan SJ, Perricone AJ, Ferris MJ, Buchwald ZS, Chowdhary M, Delman KA, Monson DK, et al: Increase in PD-L1 expression after pre-operative radiotherapy for soft tissue sarcoma. Oncoimmunology 7: e1442168, 2018

39. Lim SH, Hong M, Ahn S, Choi YL, Kim KM, Oh D, Ahn YC, Jung SH, Ahn MJ, Park K, et al: Changes in tumour expression of programmed death-ligand 1 after neoadjuvant concurrent chemoradiotherapy in patients with squamous oesophageal cancer. Eur J Cancer 52: 1-9, 2016.

40. Antonia SJ, Villegas A, Daniel D, Vicente D, Murakami S, Hui R, Yokoi T, Chiappori A, Lee KH, de Wit M, et al: Durvalumab after chemoradiotherapy in Stage III Non-Small-Cell lung cancer. N Engl J Med 377: 1919-1929, 2017.

41. Antonia SJ, Villegas A, Daniel D, Vicente D, Murakami S, Hui R, Kurata T, Chiappori A, Lee KH, de Wit M, et al: Overall survival with Durvalumab after chemoradiotherapy in Stage III NSCLC. N Engl J Med 379: 2342-2350, 2018.

42. Kwon ED, Drake CG, Scher HI, Fizazi K, Bossi A, van den Eertwegh AJ, Krainer M, Houede N, Santos R, Mahammedi $\mathrm{H}$, et al: Ipilimumab versus placebo after radiotherapy in patients with metastatic castration-resistant prostate cancer that had progressed after docetaxel chemotherapy (CA184-043): A multicentre, randomised, double-blind, phase 3 trial. Lancet Oncol 15: 700-712, 2014

43. Ludmir EB, McCaw ZR and Wei LJ: Re: Karim Fizazi, Charles G. Drake, Tomasz M. Beer, et al. Final Analysis of the Ipilimumab Versus Placebo Following Radiotherapy Phase III Trial in Postdocetaxel Metastatic Castration-resistant Prostate Cancer Identifies an Excess of Long-term Survivors. Eur Urol. In press. https://doi.org/10.1016/j.eururo.2020.07.032: Interpreting the effect of Ipilimumab following radiotherapy for patients with Postdocetaxel metastatic Castration-resistant Prostate cancer. Eur Urol 79: e10-e11, 2021.

44. Shibata A and Jeggo PA: DNA double-strand break repair in a cellular context. Clin Oncol (R Coll Radiol) 26: 243-249, 2014.

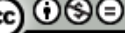

This work is licensed under a Creative Commons Attribution-NonCommercial-NoDerivatives 4.0 International (CC BY-NC-ND 4.0) License. 